\title{
Quantum Heat Engine Using Electromagnetically Induced Transparency
}

\author{
Yueyang Zou, Yue Jiang, Yefeng Mei, Xianxin Guo, and Shengwang Du* \\ Department of Physics, The Hong Kong University of Science and Technology, \\ Clear Water Bay, Kowloon, Hong Kong, China
}

(Received 18 April 2017; revised manuscript received 28 June 2017; published 4 August 2017)

\begin{abstract}
We report an experiment demonstrating the generation of directional thermal radiation with a spectral brightness that is about 9 times greater than that of the ambient pumping reservoir. The experiment is based on the recent proposal for a nontraditional quantum heat engine and uses cold $\mathrm{Rb}$ atoms, electromagnetically induced transparency, and photon correlation spectroscopy [Phys. Rev. A 94, 053859 (2016)].
\end{abstract}

DOI: 10.1103/PhysRevLett.119.050602

Electromagnetically induced transparency (EIT) [1] is a process where, by applying a strong electric field with a Rabi frequency $\Omega_{c}$ to an optically thick three-level $\Lambda$ system, the susceptibility of a weak probe is modified so as to make the medium nearly transparent at the line center of the $|1\rangle \rightarrow|3\rangle$ transition [Fig. 1(a)]. The emissive profile is also modified and typically maximizes where the absorptive profile minimizes. As recently pointed out, in such a system, Kirchhoff's law no longer applies, and, in a narrow spectral region, the radiation temperature may exceed that of a blackbody at the ambient pumping temperature [2]

In the context of quantum thermodynamics [3-8], this process may be considered as a nontraditional quantum heat engine (QHE) [2]: Here, as illustrated in Fig. 1(a), a photon is absorbed from the $|1\rangle \rightarrow|3\rangle$ reservoir at temperature $T_{13}$ and a photon is generated in the $|2\rangle \rightarrow|3\rangle$ reservoir at temperature $T_{23}$. A photon is then absorbed from the monochromatic coupling laser on the $|2\rangle \rightarrow|3\rangle$ transition, and a photon with a narrow specular and angular bandwidth is generated on the $|3\rangle \rightarrow|1\rangle$ transition. This latter radiation is the output of this engine and may drive a piston. Because its entropy is nonzero, it is in the category of low-grade work. This type of heat engine is in the class of devices suggested by Scully where nonzero off-diagonal density matrix elements lead to thermal devices with unusual properties [9-11]. For example, in Fig. 1(a), it is not required that $T_{13}>T_{23}$, and, for ideal parameters, the second law of thermodynamics allows the temperature of the generated radiation to substantially exceed that of the pumping radiation. It is the intent of this Letter to experimentally examine some predictions of Ref. [2]. Because of the very narrow generated bandwidth, for example, $0.55 \mathrm{MHz}$ as compared to the natural linewidth of $5.75 \mathrm{MHz}$, we use correlation spectroscopy and also make use of a second EIT as an ultranarrow bandpass filter.

A schematic of the overall experiment is illustrated in Fig. 1. We work with laser-cooled ${ }^{85} \mathrm{Rb}$ atoms in a dark-line two-dimensional (2D) magneto-optical trap $\left(\mathrm{MOT}_{1}\right)$ with a length of $L=1.5 \mathrm{~cm}$ [12] and an aspect ratio of about $25: 1$. Figure 1(a) shows the pertinent hyperfine levels of
${ }^{85} \mathrm{Rb}$. These are $|1\rangle=\left|5 S_{1 / 2}, F=2\right\rangle,|2\rangle=\left|5 S_{1 / 2}, F=3\right\rangle$, and $|3\rangle=\left|5 P_{1 / 2}, F=3\right\rangle$, with degeneracies $g_{1}=5$ and $g_{2}=g_{3}=7$. The atomic population spontaneous decay rates, or the Einstein $A$ coefficients, of the excited levels are $\Gamma_{31}=2 \pi \times 3.19 \mathrm{MHz}$ and $\Gamma_{32}=2 \pi \times 2.56 \mathrm{MHz}$, so that the full width at half maximum (FWHM) natural linewidth of level $|3\rangle$ is $5.75 \mathrm{MHz}$. We estimate that the residual dephasing rate of the nonallowed $|1\rangle \rightarrow|2\rangle$ transition as caused by stray magnetic fields is $\tilde{\gamma}_{21}=2 \pi \times 0.1 \mathrm{MHz}$. The experiment is run periodically, with $4.89 \mathrm{~ms}$ MOT time and $110 \mu$ s observation time in each cycle. During the observation time window, a circularly polarized $\left(\sigma^{+}\right)$ coupling laser $\left(\omega_{c}\right)$ is applied to the atomic ensemble with an angle of $3^{\circ}$ to the longitudinal $z$ axis of the $2 \mathrm{D} \mathrm{MOT}_{1}$, and four incoherent light beams (characterized by $T_{13}$ and $T_{23}$ ) are incident from $\pm 45^{\circ}$ angles.

Of importance, the Einstein $A$ coefficients of the pertinent states satisfy $\Gamma_{31}>\Gamma_{32}$. When this is the case, lasing without inversion may not occur [13], and, irrespective of parameters, with or without inversion, there is no gain within the system. There is also no phase-matching requirement of any type.

Assuming refractive indices that are sufficiently close to unity that there is negligible reflection, the spectral brightness $B(\delta, z)$ of the emission at the $|3\rangle \rightarrow|1\rangle$ transition is governed by [2]

$$
\begin{aligned}
& \frac{d B(\delta, z)}{d z}+N\left[\sigma_{\mathrm{abs}} \rho_{11}-\sigma_{\mathrm{em}}\left(\rho_{22}+\rho_{33}\right)\right] B(\delta, z) \\
& \quad=N \sigma_{\mathrm{em}}\left(\rho_{22}+\rho_{33}\right),
\end{aligned}
$$

with the boundary condition $B(\delta, z=0)=0$. Here $\delta=\omega_{13}-\omega$ is the detuning from the on-resonance transition frequency $\omega_{13}, N$ is the atomic number density, and $\rho_{i i}$ are the diagonal density matrix elements. The quantities $\sigma_{\mathrm{abs}}$ and $\sigma_{\mathrm{em}}$ are the absorption and emission cross sections, respectively, for a weak probe beam on the $|1\rangle \leftrightarrow|3\rangle$ transition $[2,14]$.

In our experimental setup [Fig. 1(b)], the thermal reservoirs at temperatures $T_{13}$ and $T_{23}$ are simulated by 


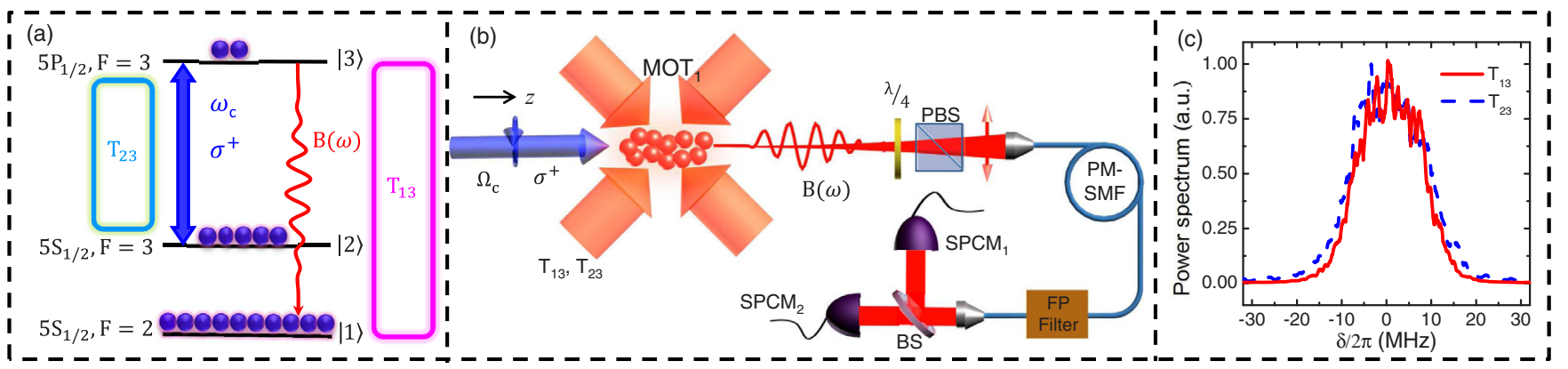

FIG. 1. Schematic of the QHE experiment. (a) Relevant atomic energy levels for the ${ }^{85} \mathrm{Rb} D 1$ line transitions. (b) The experimental setup for generating and detecting the emitted photons. The atoms prepared in a magneto-optical trap $\left(\mathrm{MOT}_{1}\right)$ are dressed by a coupling laser $\Omega_{c}$ and pumped by incoherent light beams with equivalent temperatures $T_{13}$ and $T_{23}$ on the transitions $|1\rangle \rightarrow|3\rangle$ and $|2\rangle \rightarrow|3\rangle$. The emitted photons are coupled into a polarization-maintaining single-mode fiber (PM SMF), and their intensity autocorrelation function is detected by a beam splitter (BS) and two single-photon counting modules $\left(\mathrm{SPCM}_{1}\right.$ and $\left.\mathrm{SPCM}_{2}\right)$. (c) Experimental power spectrum of the incoherent pumping sources.

incoherent light beams at $45^{\circ}$. The essential characteristic inherent to these light sources is that the phases of its spectral components are random. Each spectral component, either temporal or angular, acts independently and results in a transition rate that is linear in the incident light power. As shown in Supplemental Material [14], for a nondegenerate system, this transition rate is equal to the product of the Einstein $A$ rate and the thermal occupancy at the line center of the two-state transition. We construct such a source by modulating a narrow-band $(<1 \mathrm{MHz})$ laser beam with an acousto-optic modulator that itself is driven by white noise $[15,16]$ from a (Tektronix AFG3252) function generator [17]. Figure 1(c) shows the power spectrum of the two incoherent light sources $\left(T_{13}\right.$ and $\left.T_{23}\right)$ with a FWHM of $20 \mathrm{MHz}$ (wide as compared to the natural linewidth of $5.75 \mathrm{MHz}$ ). The emitted photons along the longitudinal $z$ axis pass though a $\sigma^{+}$polarization selector, consisting of a $\lambda / 4$ wave plate and a polarization beam splitter and are coupled into a polarization-maintaining (PM) single-mode fiber (SMF). A Fabry-Perot cavity filter with a bandwidth of $500 \mathrm{MHz}$ is used to eliminate the scattered light from the coupling laser beam.

In order to determine the equivalent temperatures of the incoherent sources, we make use of the relations

$$
\begin{aligned}
& R_{13}=\frac{g_{3}}{g_{1}} \Gamma_{31} \bar{n}_{13}=\frac{g_{3}}{g_{1}} \Gamma_{31}\left\{\exp \left[\hbar \omega_{13} / k_{b} T_{13}\right]-1\right\}^{-1}, \\
& R_{23}=\frac{g_{3}}{g_{2}} \Gamma_{32} \bar{n}_{23}=\frac{g_{3}}{g_{2}} \Gamma_{32}\left\{\exp \left[\hbar \omega_{23} / k_{b} T_{23}\right]-1\right\}^{-1},
\end{aligned}
$$

where $k_{b}$ is the Boltzmann constant and $\bar{n}_{i j}=$ $\left\{\exp \left[\hbar \omega_{i j} / k_{b} T_{i j}\right]-1\right\}^{-1}$ is the thermal occupation number of the $|i\rangle \rightarrow|j\rangle$ transition. The quantities $R_{i j}$ are the transition pumping rates that result from the incoherent laser sources when each is applied independently. With the transition frequencies $\omega_{i j}$ known, the measured $R_{i j}$ determine the effective temperatures $T_{i j}$ and occupancy numbers $\bar{n}_{i j}$ of the respective sources.

To measure the pumping rate $R_{13}$, we turn off both the coupling laser and the incoherent light source at $T_{23}$. We measure the optical depth (OD) of the $|1\rangle \rightarrow|3\rangle$ transition as a function of time in the presence of the $T_{13}$ source. With $N_{1}(t)=N \rho_{11}(t)$ equal to the ground state population and using $\operatorname{OD}(t)=N_{1}(t) \sigma_{\text {abs }} L$, we obtain $N_{1}(t)$ and extract the pumping rate $R_{13}$ by fitting the solution of the three-level rate equations to $N_{1}(t)$ (see Supplemental Material [14]). The red circles in Fig. 2 show the measured pumping rate $R_{13}$ as a function of the pumping power. Using Eq. (2), we convert these measured pumping rates to values of equivalent temperature $T_{13}$ (square points). The red dashed line is the best linear fit to the dependence of $R_{13}$ on the pumping power. The solid blue line is calculated from the red dashed line using Eq. (2). In a similar way, by measuring the pumping rate $R_{23}$, we calibrate the equivalent temperature $T_{23}$.

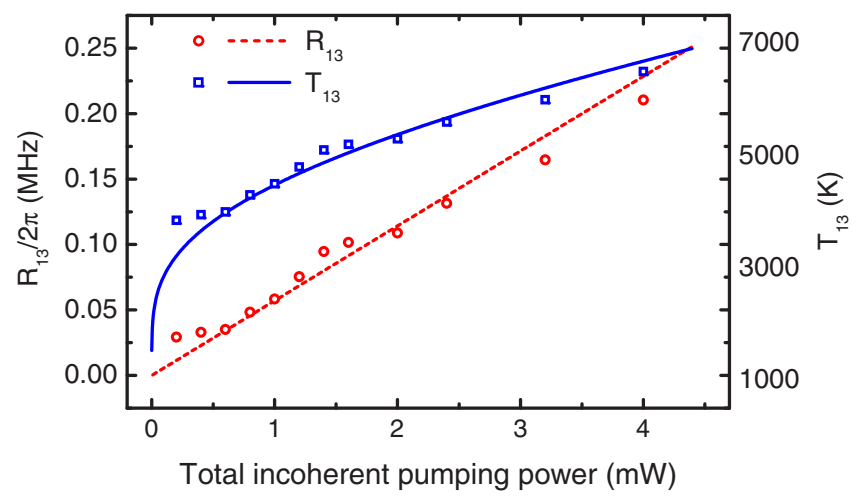

FIG. 2. Pumping rate $R_{13}$ and temperature $T_{13}$ as functions of the incoherent pumping light power. The (red) circle points are the measured $R_{13}$, and the (red) dashed line is the best linear fit. The (blue) square points are the temperature $T_{13}$ corresponding to the values of $R_{13}$ calculated from Eq. (2). The (blue) solid line is calculated from the dashed line. 


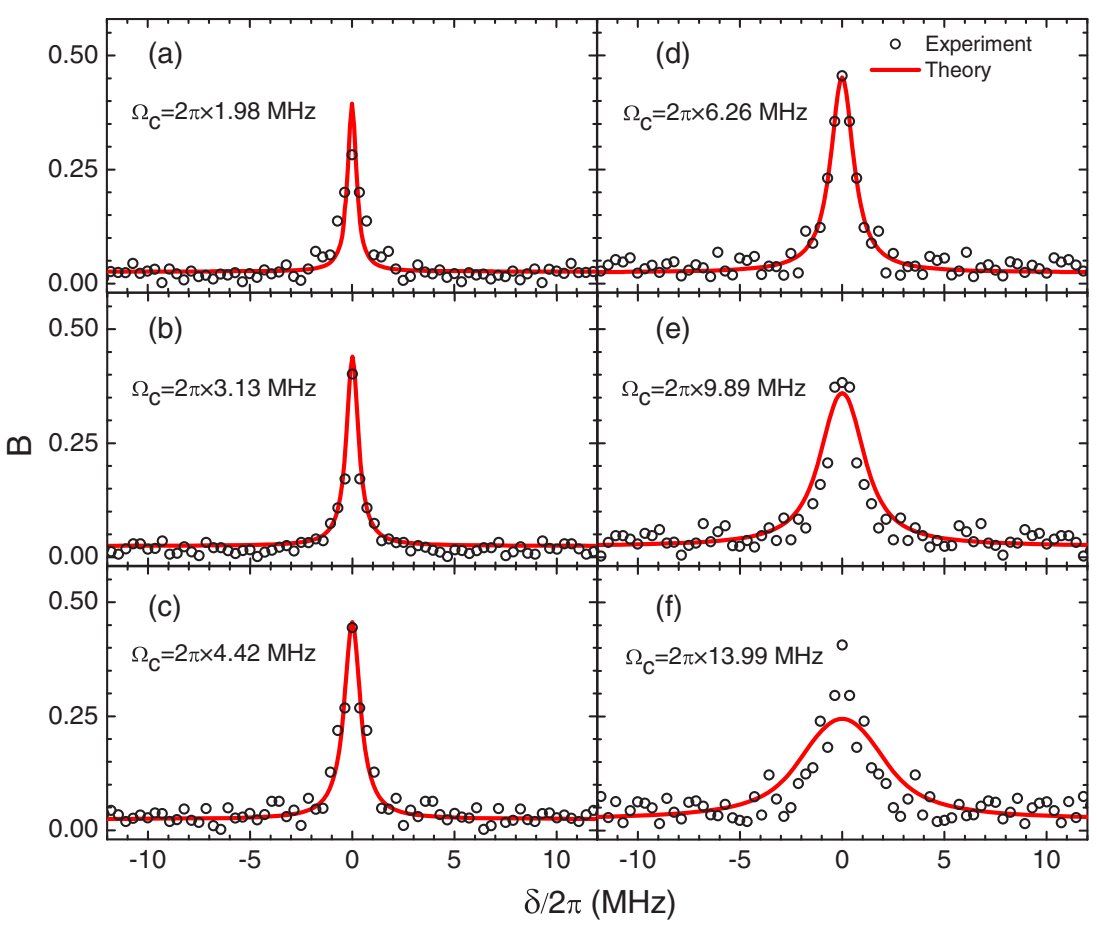

FIG. 3. The power spectrum $B(\delta)$ of generated photons at $T_{13}=T_{23}=5000 \mathrm{~K}$ and $\mathrm{OD}_{0}=105$ under different coupling laser powers. The FWHM bandwidths of the photons are (a) 0.55 , (b) 0.75 , (c) 1.00, (d) 1.41, (e) 2.73, and (f) $5.27 \mathrm{MHz}$. The (red) solid lines are the theoretical curves. The (black) circles (experimental data) are obtained from the Fourier transform of $\sqrt{g^{(2)}}(\tau)-1$ with arbitrary vertical scaling for the best fit to the theory. The corresponding $g^{(2)}(\tau)$ are plotted in Fig. S2 of Supplemental Material [14].

The first objective of this experiment is to examine the line shape of the emissive spectrum and, in particular, to verify that it may be narrower than the natural linewidth. In order to do this, we use a beam splitter and singlephoton counting modules, as shown in Fig. 1(b), to measure the normalized intensity correlation function $g^{(2)}(\tau)=$ $\langle I(t) I(t+\tau)\rangle /\langle I(t)\rangle^{2}$, where $I(t)$ is the intensity. Assuming chaotic light (arbitrary spectral phases), the spectrum of the emitted radiation is obtained from $B(\delta)=A \mathcal{F}\left\{\sqrt{g^{(2)}(\tau)-1}\right\}$. Here $\mathcal{F}$ denotes the Fourier transform, and $A$ is an arbitrary scaling factor. The results under the conditions $T_{13}=T_{23}=5000 \mathrm{~K}$ and $\mathrm{OD}_{0}=$ $N \sigma_{0} L=105$ at different coupling laser $\Omega_{c}$ strengths are displayed in Fig. 3 where $\sigma_{0}=\left(\lambda^{2} / 2 \pi\right)\left(\Gamma_{31} / \Gamma_{3}\right)\left(g_{3} / g_{1}\right)$ with $\Gamma_{3}=\Gamma_{31}+\Gamma_{32}$ [The corresponding $g^{(2)}(\tau)$ are plotted in Fig. S2 of Supplemental Material [14].] The solid lines are the theoretical curves of $B(\delta)$ calculated from Eq. (1). The black circular points are the experimental data. Each experimental plot is scaled independently for the best fit to the theory. It is clear that, as predicted by the theory, the photon bandwidth reduces as the coupling laser power is reduced. At $\Omega_{c}=2 \pi \times 1.98 \mathrm{MHz}$ [see Fig. 3(a)], the bandwidth of the emitted photons is $0.55 \mathrm{MHz}$ and is narrower than the natural linewidth by a factor of about 10 .

We now turn to the measurement of the spectral brightness of the emission. Because the emissive and absorptive profiles are not the same, the theory predicts [2] that over a narrow spectral region the ratio of the on-line center heat engine emission to the emission of a blackbody in the absence of EIT should exceed unity, i.e., $B(0) / \bar{n}_{13} \geq 1$. To measure this ratio, we construct a narrow-band filter by using a second ${ }^{85} \mathrm{Rb}$ MOT $\left(\mathrm{MOT}_{2}, \mathrm{OD}=33\right)$, as shown in Fig. 4(a). Figure 4(b) shows the transmission function of this filter with and without a coupling laser present at $\mathrm{MOT}_{2}$. We term these filter functions as $H_{1}(\delta)$ and $H_{0}(\delta)$, respectively, and adjust the strength of the coupling laser to set the width of the transmission window $H_{1}(\delta)$ at $2.8 \mathrm{MHz}$.

To compare the theory and experiment, we define the normalized average emissivity $\overline{\mathcal{E}}$ as

$$
\overline{\mathcal{E}}=\frac{B_{T}}{n_{T}}=\frac{\int_{-\infty}^{\infty} B(\delta)\left[H_{1}(\delta)-H_{0}(\delta)\right] d \delta}{\bar{n}_{13} \int_{-\infty}^{\infty}\left[H_{1}(\delta)-H_{0}(\delta)\right] d \delta} .
$$

$B_{T}$ is the filtered brightness that passes through the EIT filter. The quantity $n_{T}$ is the corresponding contribution from the blackbody radiation that passes through this filter. We obtain $B_{T}$ by measuring the photon rate difference with and without EIT at $\mathrm{MOT}_{2} . n_{T}$ is obtained in the same way by turning off the coupling laser in the QHE $\left(\mathrm{MOT}_{1}\right)$. This process helps to remove the effects of stray noise counts and detector dark counts on $B_{T}$ and $n_{T}$. The results for the normalized emissivity $\overline{\mathcal{E}}$ as a function of the coupling Rabi 
(a) $s_{0}$

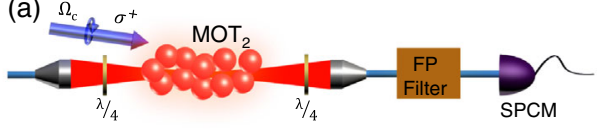

(b)

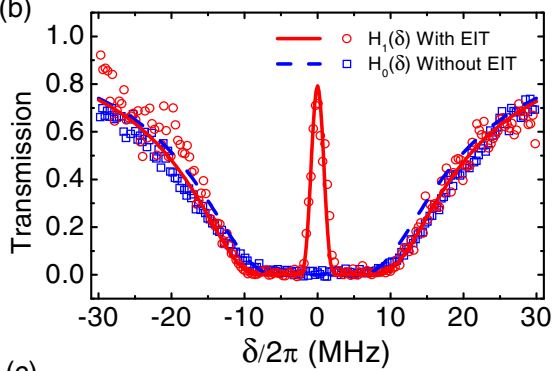

(c)

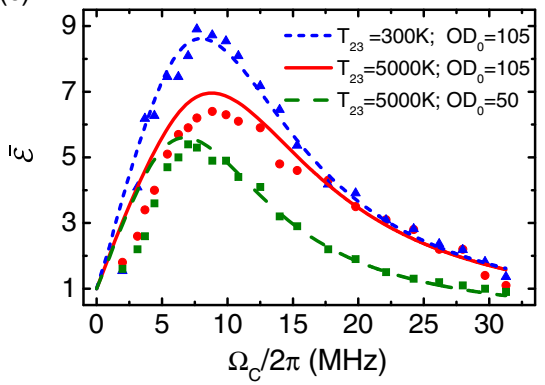

FIG. 4. (a) Experimental setup for measuring the relative spectral brightness of the photon emission. The generated photons pass through a narrow-bandwidth EIT filter at $\mathrm{MOT}_{2}$ before detection by the SPCM. (b) The transmission profiles of $\mathrm{MOT}_{2}$ with and without EIT. (c) The averaged relative spectral brightness $\overline{\mathcal{E}}$ as a function of the coupling laser Rabi frequency $\Omega_{c} . T_{13}=5000 \mathrm{~K}$ is fixed for all the measurements. The square (green), circle (red), and triangle (blue) points are experimental data. The dashed (green), solid (red), and dotted (blue) curves are the theoretical plots from Eq. (3).

frequency $\Omega_{c}$ are plotted in Fig. 4(c) with the squares, circles, and triangles denoting the different experimental conditions. Of importance, there are no fitting parameters for the theoretical curves calculated from Eq. (3). The temperature of the $T_{13}$ incoherent source is $5000 \mathrm{~K}$ for each of the three plots. $T_{23}$ and the optical depth are varied as shown. In each of the three plots, $\overline{\mathcal{E}} \rightarrow 1$ as $\Omega_{c} \rightarrow 0$. For $T_{23}=300 \mathrm{~K}$ (room temperature), the peak increase of $\overline{\mathcal{E}}$ is 9. This may be compared with a theoretical prediction of the normalized emissivity $B(0) / \bar{n}_{13}=19$ as the filter bandwidth is reduced to zero [14]. The overall shape of the curves in Fig. 4(c) may be explained as follows: First, the essence of the enhancement of the emissivity is the result of quantum interference. As the coupling laser is increased from zero, the atomic system is dressed so as to result in a destructive interference in the absorption cross section and, at the same time, a constructive increase in the cross section for emission; see Fig. 2 of Ref. [2]. The emissivity therefore exceeds unity, and Kirchhoff's law is no longer valid. At a fixed optical depth and higher Rabi frequencies, the absorption from the reservoirs is reduced because of the wider EIT bandwidth, and the generated brightness approaches that of the background blackbody radiation.

We remark on the use of the second law of thermodynamics: In Ref. [2], it was shown that when $T_{23}>T_{13}$ and when $\Gamma_{32}>\Gamma_{31}$ the second law may be used to predict and limit the allowed increase in emissivity. When $T_{13}>T_{23}$ the second law does not limit this increase [14]. If the extraneous residual dephasing rate $\tilde{\gamma}_{21}$ is set to zero, we find numerically that the enhancement factor increases from 19 to more than 100. Of importance, the condition $\Gamma_{31}>\Gamma_{32}$ ensures that the enhancement described here is not a result of gain on the $|1\rangle \rightarrow|3\rangle$ transition.

In summary, we have shown that EIT allows the spectral brightness of a radiating blackbody to have a line shape that is narrower and a peak intensity that is greater than that of a blackbody at the ambient pumping temperature. The system is symmetric in the forward $(+z)$ and backward $(-z)$ directions, confirmed by the EIT measurements in both directions [14]. These results show a violation of detailed balance and Kirchhoff's law and may also be viewed as an early demonstration of a nontraditional coherence-based quantum heat engine [18].

The authors thank S. E. Harris for helpful discussions and contributions to the manuscript. S. D. acknowledges the JILA Visiting Fellows Program during his stay at JILA, University of Colorado Boulder. The work was supported by the Hong Kong Research Grants Council (Project No. 16305615) and in part by the CAS/SAFEA International Partnership Program for Creative Research Teams.

Y.Z. and Y. J. contributed equally to this work.

*Corresponding author. dusw@ust.hk

[1] S. E. Harris, Electromagnetically induced transparency, Phys. Today 50, No. 7, 36 (1997).

[2] S. E. Harris, Electromagnetically induced transparency and quantum heat engines, Phys. Rev. A 94, 053859 (2016).

[3] J. Roßnagel, S. T. Dawkins, K. N. Tolazzi, O. Abah, E. Lutz, F. Schmidt-Kaler, and Kilian Singer, A single-atom heat engine, Science 352, 325 (2016).

[4] R. Kosloff and A. Levy, Quantum heat engines and refrigerators: Continuous devices, Annu. Rev. Phys. Chem. 65, 365 (2014).

[5] H. E. D. Scovil and E. O. Schulz-DuBois, Three-Level Masers as Heat Engines, Phys. Rev. Lett. 2, 262 (1959).

[6] J. E. Geusic, E. O. Schulz-DuBois, and H. E. D. Scovil, Quantum equivalent of the carnot cycle, Phys. Rev. 156, 343 (1967).

[7] H. T. Quan, Y.-X. Liu, C. P. Sun, and F. Nori, Quantum thermodynamic cycles and quantum heat engines, Phys. Rev. E 76, 031105 (2007).

[8] D. Gelbwaser-Klimovsky, R. Alicki, and G. Kurizki, Minimal universal quantum heat machine, Phys. Rev. E 87, 012140 (2013). 
[9] M. O. Scully, Extracting Work from a Single Thermal Bath via Quantum Negentropy, Phys. Rev. Lett. 87, 220601 (2001).

[10] M. O. Scully, M. S. Zubairy, G. S. Agarwal, and H. Walther, Extracting work from a single heat bath via vanishing quantum coherence, Science 299, 862 (2003).

[11] M. O. Scully, Quantum Photocell: Using Quantum Coherence to Reduce Radiative Recombination and Increase Efficiency, Phys. Rev. Lett. 104, 207701 (2010).

[12] S. Zhang, J. F. Chen, C. Liu, S. Zhou, M. M. T. Loy, G. K. L. Wong, and S. Du, A dark-line two-dimensional magnetooptical trap of $85 \mathrm{Rb}$ atoms with high optical depth, Rev. Sci. Instrum. 83, 073102 (2012).

[13] A. Imamoğlu, J. E. Field, and S. E. Harris, Lasers without Inversion: A Closed Lifetime Broadened System, Phys. Rev. Lett. 66, 1154 (1991).

[14] See Supplemental Material at http://link.aps.org/ supplemental/10.1103/PhysRevLett.119.050602 for (i) a discussion of thermal-like excitation by incoherent pumping, (ii) formulas for the absorption and emission cross sections, (iii) details of pumping rate measurements, (iv) intensity correlation function $g^{(2)}(\tau)$, (v) the theoretical plots of relative spectral brightness $B(0) / \bar{n}_{13}$ vs the coupling Rabi frequency, (vi) the second law of thermodynamics, and (vii) EIT measurements in both the forward and backward directions.

[15] G. G. Padmabandu, G. R. Welch, I. N. Shubin, E. S. Fry, D. E. Nikonov, M. D. Lukin, and M. O. Scully, Laser Oscillation without Population Inversion in a Sodium Atomic Beam, Phys. Rev. Lett. 76, 2053 (1996).

[16] M. Fleischhauer, M. D. Lukin, D. E. Nikonov, and M. O. Scully, Influence of pump-field phase diffusion on laser gain in a double- $\Lambda$ non-inversion laser, Opt. Commun. 110, 351 (1994).

[17] We note that, though this incoherent source results in a linear transition rate and therefore satisfies the requirements of this work, its normalized second-order correlation function $g^{(2)}(0)=1$ and is not equal to that of a true blackbody source where $g^{(2)}(0)=2$. Here the normalized second-order correlation function of a light source with intensity $I(t)$ is defined as $g^{(2)}(\tau)=\langle I(t) I(t+\tau)\rangle /\langle I(t)\rangle^{2}$.

[18] D. A. B. Miller, L. Zhu, and S. Fan, Universal modal radiation laws for all thermal emitters, Proc. Natl. Acad. Sci. U.S.A. 114, 4336 (2017). 